\title{
Clinical Evaluation of Irreversible Data Compression for Computed Radiography in Excretory Urography
}

\author{
Kazuhiko Uchida, Katsumi Nakamura, Hideyuki Watanabe, Kanji Egashira, Yoichi Ishino, \\ Minoru Murakami, and Hajime Nakata
}

\begin{abstract}
Efficient data compression is required for practical daily use of digital images in computed radiography (CR). This study investigated the clinical utility of data compression in excretory urography using the FCR 9000 system (Fuji Photo Film, Tokyo, Japan). Type III data compression technique was used, which can achieve a 20:1 to 25:1 compression ratio. To evaluate the degradation of image quality, we compared paired original and compressed CR images. Although a slight deterioration of image quality was noted in the renal calyx including collecting tubules among various anatomical structures, the difference was not significant. Receiver operating characteristics analysis revealed no significant difference among the original and compressed images. We conclude that compressed CR images using Type III data compression technique in excretory urography appear to be clinically applicable and acceptable.
\end{abstract}

Copyright (C) 1996 by W.B. Saunders Company

KEY WORDS: computed radiography (CR), data compression, excretory urography.

COMPUTED radiography (CR) has currently become an important technology replacing screen-film system for conventional radiographs. Digital data used in CR are superior to conventional analog radiographs for image transfer and storage. One image using CR requires usually a $1,770 \times 1,770$ or 2,150 matrix with 10 bits and large quantities of data are produced daily by various radiographic examinations in any hospital. It is not practical to use these originally produced data for transfer and storage. An effective and useful data compression technique seems to be necessary for daily clinical operation of a CR system.

Recent studies suggest that a high compression ratio of at least 20:1 is achievable without significantly affecting observer performance in digital chest radiographs. ${ }^{1-3} \mathrm{We}$ also previously reported the clinical applicability of a high data compression in chest $^{4}$ and gastrointestinal CR examinations. ${ }^{5}$ A FCR system (Fuji Photo Film, Tokyo, Japan) offers three types of data compression. In this study, we evaluated the clinical utility of Type III data compression technique in excretory urography using this CR system. This compression technique is based on prediction and Huffman coding and achieves an ap- proximately $20: 1$ to $25: 1$ compression ratio. The characteristics of this technique are as follows: (1) efficient predictions can be made using interpolation prediction from the main data, and from this, a high compression ratio can be achieved; (2) contrast resolution is compressed by shift-quantification without decreasing the spatial resolution; (3) all data are organized hierarchically to facilitate coding of the data base. In addition, this compression technique has the advantage of removing block artifacts that may appear between blocks of high and low activity when using a two-dimensional discrete cosine transform compression method. However, the compression ratio is not constant using the current technique, because the volume of omitted data depend on picture elements in each image. ${ }^{4}$

\section{MATERIALS AND METHODS}

The FCR 9000 system offers three types of data compression and we selected the Type III data compression technique. This is an irreversible technique, but it is capable of a high 20:1 to $25: 1$ compression ratio.

CR images for excretory urography were obtained using ST-V imaging plates $(35 \mathrm{~cm} \times 43 \mathrm{~cm}, 1,770 \times 2,150$ matrix, 10 bits, pixel size $200 \mu \mathrm{m}$ ). Image reading of the CR data was performed with an automatic exposure recognition method and the image processing was performed with a standard setting. Hard copy images of $23.5 \mathrm{~cm} \times 28.5 \mathrm{~cm}$ were produced using a laser printer (FL-IMD, Fuji Photo Film). Exposure factors were $66 \mathrm{KV}$ and $48 \mathrm{mAs}$ with a $10: 1$ grid for adults. Excretory urography was performed either with an intravenous bolus injection of $50 \mathrm{~mL}$ of $65 \%$ iohexol (intravenous pyelography, [IVP]) or with a drip infusion of $100 \mathrm{~mL}$ of $65 \%$ iohexol (drip infusion pyelography, [DIP]) The IVP images were obtained at 5 and 15 minutes, whereas DIP images were obtained immediately and 10 minutes after the completion of the administration of the contrast material in addition to the scout images.

First we evaluated the possibility of the image degrada-

From the Department of Radiology, University of Occupational and Environmental Health, Yahatanishi-ku, Kitakyushushi, Japan.

Address reprint requests to Hajime Nakata, MD, Department of Radiology, University of Occupational and Environmental Health, Yahatanishi-ku, Kitakyushu-shi 807, Japan.

Copyright 1996 by W.B. Saunders Company

0897-1889/96/0903-0008\$3.00/0 
tion by compression on two types of cases, normal urography and urinary stone, by visually comparing the original and compressed images. Twenty cases of normal urography (10 IVP, 10 DIP), were selected. These were comprised of 10 males and 10 females with ages ranging from 6 to 85 years (mean: 49.8 years). For urinary stone, 11 cases consisting of 3 renal, 7 ureteral and 1 bladder stone (7 IVP, 4 DIP) were used. Their greatest diameters ranged from $2 \mathrm{~mm}$ to $9 \mathrm{~mm}$ (mean: $5 \mathrm{~mm}$ ). There were 4 males and 7 females with ages ranging from 20 to 74 years (mean: 53.7 years).

A pair of hard copies of original images and images reconstructed using Type III compression technique were made for each case. For normal excretory urography only the images with the contrast material were used. For urinary stone, the scout images were included. The paired images were presented in tandem on a view box to five certified radiologists (KN, HW, KE, YI, and MM). They were not told which one of the pair was the original or compressed image and were asked to compare the paired images as follows; (1) the top image definitely superior to the bottom image, (2) the top image superior to the bottom image, (3) the top image equal to the bottom image, (4) the bottom image superior to the top image, (5) the bottom image definitely superior to the top image. Normal cases were evaluated as for nephrogram, calyx including collecting tubules, renal pelvis to ureter, and urinary bladder. For cases of urinary stones, observation was concentrated only on the stones (Figs 1 and 2). Responses for both urinary stones and normal cases were translated as follows; (1) original definitely superior to compressed image, (2) original superior to compressed image, (3) original equal to compressed image, (4) compressed image superior to original, (5) compressed image definitely superior to original. Statistical evaluation was performed using the $\chi^{2}$ test.

To evaluate the effects of compression on diagnostic accuracy in excretory urography, we also analyzed observer performance by receiver operating characteristic (ROC)

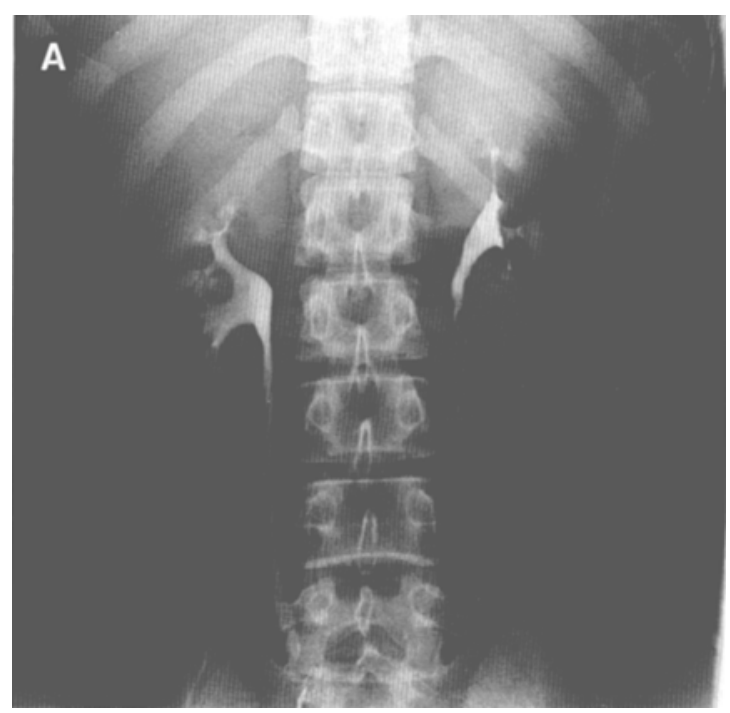

analysis. Thirty true-negative (15 IVP, 15 DIP, 18 males and 12 females, 6 to 85 years, mean: 53.2 years) and 20 true-positive cases (12 IVP, 8 DIP, 11 males and 9 females, 20 to 86 years, mean: 54.3 years) were used. Included were 9 urinary stones, 4 renal atrophy, 2 hydronephrosis, 1 renal cell carcinoma, 1 renal pelvic transitional cell carcinoma, 1 renal cyst, 1 urinary bladder carcinoma, and 1 renal arteriovenous malformation. The criteria for selecting the abnormal cases were (1) the size of the urinary stones less than 10 $\mathrm{mm}$, and (2) variety of abnormalities clearly visible on original CR images (Figs 3 and 4). Final diagnoses were established by pathological findings, other diagnostic procedure or clinical follow-up. The same five radiologists again participated in this study and the observation was first performed using sets of compressed scout and contrast CR images. At least 1 week thereafter, the test was repeated on sets of original CR images. For each set of images, the observers indicated the presence or absence of the radiographic abnormality in question on continuously distributed data from 0 to 100 , with 0 indicating definite absence and 100 definite presence of the abnormality. A maximum of 30 seconds was allowed for viewing each CR image. For a comparison of paired ROC data, the area under ROC curves (Az) was calculated from each ROC curve using the software "LABROC" (Metz CE, University of Chicago, IL). Statistical evaluations were performed by paired $t$-test.

\section{RESULTS}

Results comparing compressed images with the originals are shown in Table 1 . None of the radiologists judged "original definitely superior to compressed image" in any pair. For normal anatomical structures, the majority of pairs were judged "original equal to compressed image" ranging from a low of $72 \%$ for renal

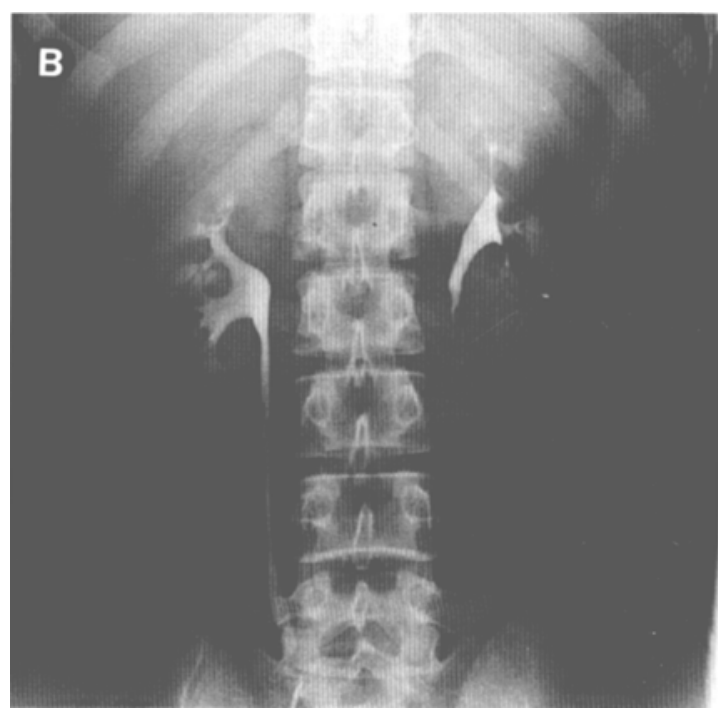

Fig 1. Normal IVP with nephrogram, renal calyx, renal pelvis, and ureter. (A) Original image. (B) Compressed image. There was only a slight degradation in the visualization of renal calyx. 

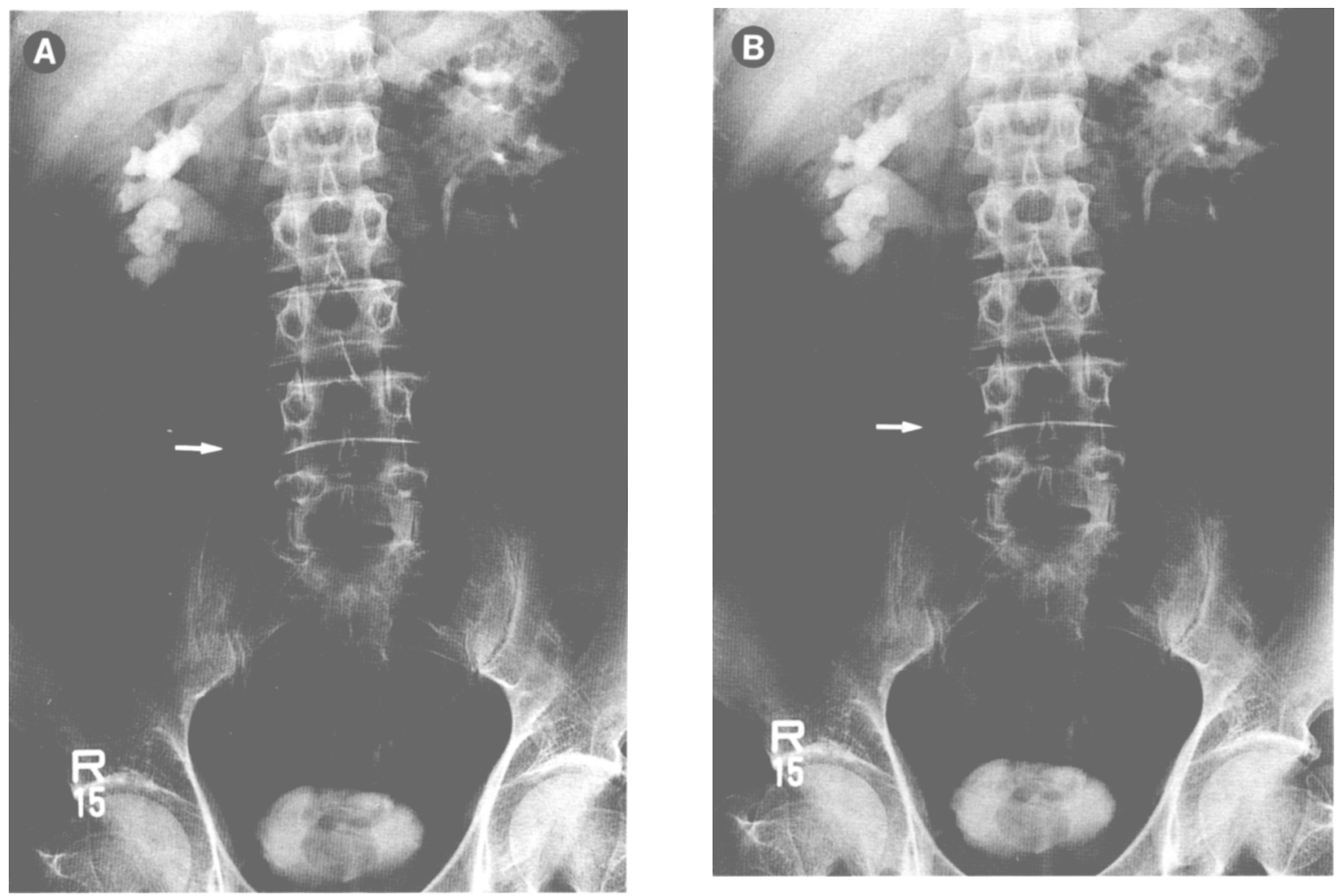

Fig 2. IVP with right ureteral stone, $4 \mathrm{~mm}$ in diameter (arrow) causing hydronephrosis. (A) Original image. (B) Compressed image. There was no degradation of the image quality.

calyx to a high of $100 \%$ for urinary bladder. The original images were judged superior to compressed images in $27 \%$ for calyx. As for urinary stones, $25 \%$ were judged "original superior to compressed image" but none of the pairs were judged "original definitely superior to com-

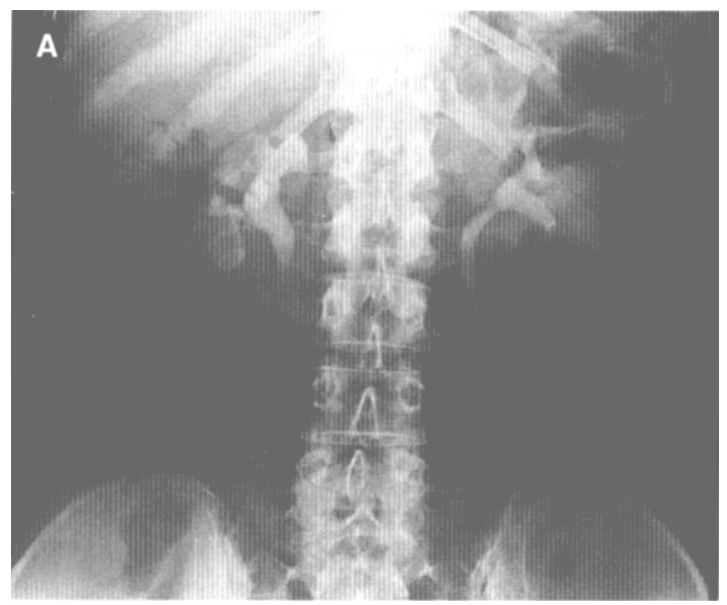

pressed image." In all cases, the stones could be detected on compressed images. Overall, the difference between compressed and original images was not significant at $5 \%$ of significant level.

ROC curves obtained are shown in Fig 5. The

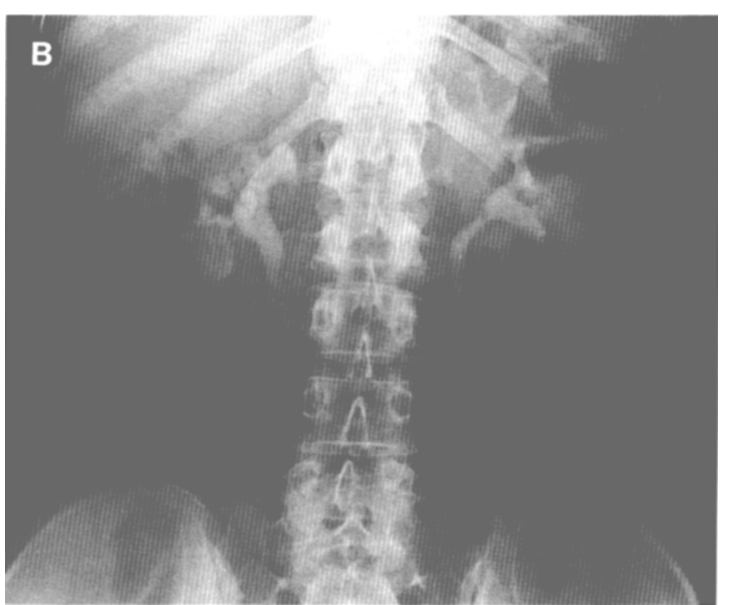

Fig 3. IVP with right renal atrophy. (A) Original image. (B) Compressed image. The renal outline and deformed renal calyces were recognized and there was no diagnostic problem. 

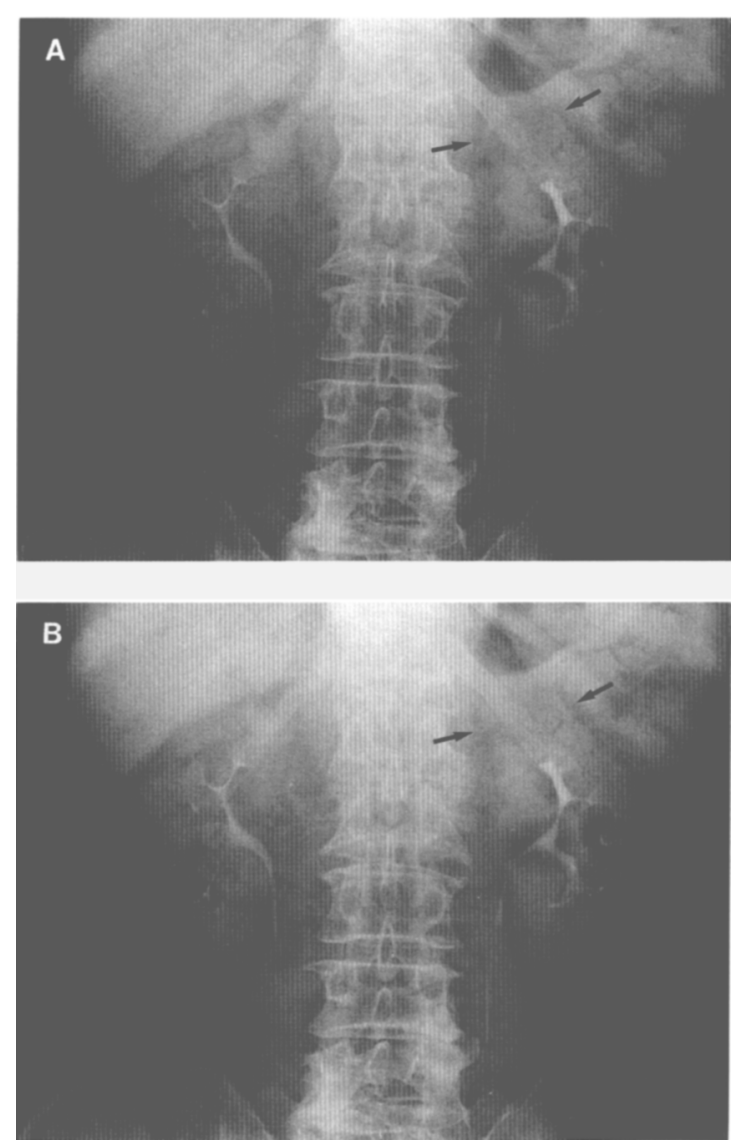

Fig 4. DIP with left renal cyst (arrows). (A) Original image. (B) Compressed image. There was no problem in pointing out the lesion.

areas under the ROC curves $(\mathrm{Az})$, which represent the probability of diagnostic accuracy ${ }^{6.7}$ were $0.869 \pm 0.058$ for original images and $0.861 \pm 0.057$ for compressed images on average. The difference was not significant at $5 \%$ of significant level.

Table 1. Comparison between Compressed and Original CR Image in Normal Urography and Urinary Stone

\begin{tabular}{cccccc}
\hline & $\begin{array}{c}\text { Nephro- } \\
\text { gram (\%) }\end{array}$ & $\begin{array}{c}\text { Calyx } \\
(\%)\end{array}$ & $\begin{array}{c}\text { Renal Pelvis } \\
\text { to Ureter } \\
(\%)\end{array}$ & $\begin{array}{c}\text { Bladder } \\
(\%)\end{array}$ & $\begin{array}{c}\text { Urinary Stone } \\
(\%)\end{array}$ \\
\hline 1 & 0 & 0 & 0 & 0 & 0 \\
2 & 7 & 27 & 1 & 0 & 25 \\
3 & 93 & 72 & 98 & 100 & 71 \\
4 & 0 & 1 & 1 & 0 & 4 \\
5 & 0 & 0 & 0 & 0 & 0 \\
\hline
\end{tabular}

1, Original definitely superior to compressed image: 2 , Original superior to compressed image; 3 , Originat equal to com. pressed image: 4, Compressed image superior to origina!: 5 , Compressed image definitely superior to original.

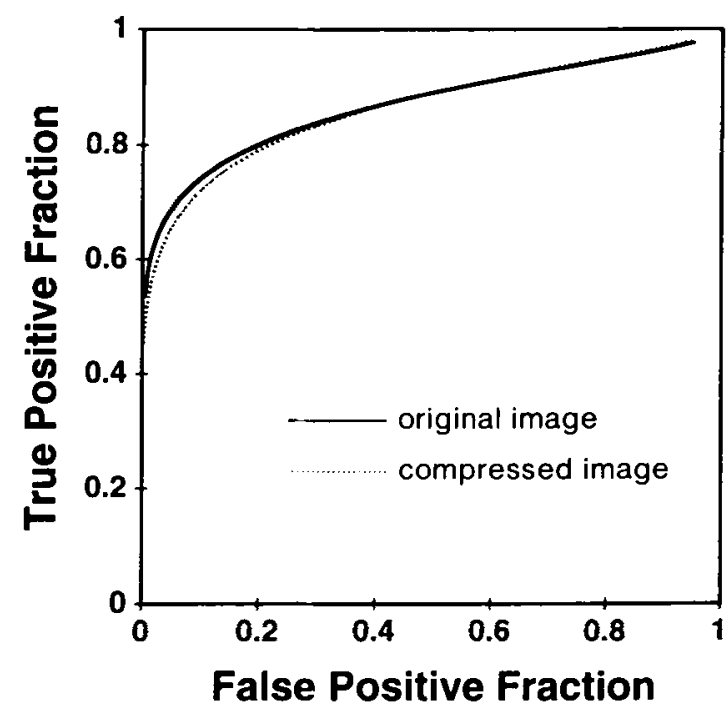

Fig 5. ROC curves for original and compressed images. Az for original images: $0.869 \pm 0.058 \mathrm{Az}$, for compressed images: $0.861 \pm 0.057$. There was no difference between the two at $5 \%$ significant level.

\section{DISCUSSION}

Although the tendency toward deterioration of image quality was identified on the compressed images, there was no significant difference between compressed and original images. Because contrast material is used in excretory urography, the target of observation is pronounced and therefore the effect of compression can be minimized. However, the renal calyx tended to show a slight loss of image quality. The calyx often includes visualization of collecting tubules with fine, sharp structures and the differences in density on the original x-ray image are subtle and this may be affected by compression.

Calcification can also be degraded on compressed images. This is probably because an imaging plate of $200 \mu \mathrm{m}$ pixel size was used in this CR system and the $2 \times 2$ pixel subsampling in the first process of compression means averaging 4 pixels, which may make the contour unsharp. A microcalcification on CR mammography is most sensitive to the degradation of image quality. ${ }^{8}$ Although urinary stones showed a slight deterioration on compressed images in the current study, no significant difference was found between the compressed and original images. The smallest stone in this study was 2 
$\mathrm{mm}$ in diameter. This means there is probably no problem in the observation of gross calcifications encountered in ordinary urinary stones. However, a possibility remains that small calcific stones might not be detected on compressed images.

The second evaluation method, ROC analysis of clinical cases also showed no significant difference between compressed and original images. This confirms that Type III compres- sion does not appear to affect diagnostic accuracy in excretory urography. We conclude that this compression technique is clinically applicable for CR excretory urography. Its use in daily practice will facilitate the transfer and storage of excretory urography images.

\section{ACKNOWLEDGMENT}

We are grateful to Charles E. Metz, MD, for permitting the use of software (LABROC).

\section{REFERENCES}

1. MacMahon H, Doi K, Sanada S, et al: Data compression: Effect on diagnostic accuracy in digital chest radiography. Radiology 178:175-179, 1991

2. Ishigaki T, Sakuma S, Ikeda M, et al: Clinical evaluation of irreversible image compression: Analysis of chest imaging with computed radiography. Radiology 175:739743,1990

3. Goldberg MA, Pvovarov M, Mayo-Smith WW, et al: Application of wavelet compression to digitized radiographs. Am J Roentgenol 163:463-468, 1994

4. Mori T, Nakata H: Irreversible data compression in chest imaging using computed radiography; An evaluation. J Thorac Imag 9:23-30, 1994
5. Uchida K: Evaluation of data compression in gastrointestinal examinations using computed radiography. Nippon Act Radiol (in press, Japanese)

6. Hanley JA, McNeil BJ: The meaning and use of the area under a receiver operating characteristic (ROC) curve. Radiology 143:29-36, 1982

7. Metz CE: ROC methodology in radiologic imaging. Invest Radiol 21:720-733, 1986

8. Murakami S, Oda N, Terada K, et al: Data compression for chest radiography and mammography using computed radiography. Jpn J Radiol Technol 51:13-18, 1995 (Japanese) 